\title{
Thoracoscopic Repair of Recurrent Bochdalek Diaphragmatic Hernias in Children
}

\author{
Shaun M. Kunisaki, MD, Ian A. Powelson, Steven W. Bruch, MD, Marcus D. Jarboe, MD, \\ James D. Geiger, MD, and George B. Mychaliska, MD
}

\begin{abstract}
Background: Recurrent herniation is a well-known complication following the initial repair of congenital diaphragmatic hernias (CDHs). The role of minimally invasive surgical techniques in recurrent $\mathrm{CDH}$ remains undefined. The purpose of this study was to evaluate our early experience with thoracoscopic repair compared with traditional open repair in children with recurrent $\mathrm{CDH}$.

Subjects and Methods: We retrospectively reviewed all recurrent Bochdalek CDH cases $(n=24)$ managed at a single tertiary-care referral center between January 1990 and March 2011. Children who underwent thoracoscopic repair for recurrent $\mathrm{CDH}$ were identified, and their data were compared by the unpaired $t$ test and the two-sided Fisher's exact test, as appropriate, with those of children who underwent open repair. Significance was defined as $P<.05$.

Results: Thoracoscopic repair was attempted in $6(25 \%)$ children with recurrent CDH. Four $(67 \%)$ repairs were successfully completed without conversion to an open procedure. The mean age at thoracoscopic repair was 11.5 months (range, 8.1-16.1 months). The mean operative time was 191 minutes (range, 94-296 minutes), and all children were extubated within 24 hours. The mean hospital length of stay was 3.75 days (range, 1-6 days). There were no deaths or subsequent recurrences after a mean follow-up of 26.5 months (range, 14.3-41.3 months). There were no statistical differences in any of the measured outcome variables when compared with the open repair group.

Conclusions: Our initial experience suggests that thoracoscopic repair is a feasible alternative to open repair in selected children with recurrent Bochdalek diaphragmatic hernias.
\end{abstract}

\section{Introduction}

$\mathbf{H}$ ERNIA RECURRENCE is a well-known complication following the initial repair of posterolateral (Bockdalek) congenital diaphragmatic hernias (CDHs). ${ }^{1}$ Based on reports from several major centers worldwide, the incidence of recurrent herniation ranges from $4 \%$ to $50 \%$ among $\mathrm{CDH}$ survivors. $^{2-6}$ The vast majority of these recurrences occur within the first few years of life. ${ }^{7}$ Several different operative approaches, including the use of muscle flaps and novel bioprotheses, have been described in an attempt to solve this problem. ${ }^{3,8-10}$ Unfortunately, none of these techniques has been shown to be superior to patch repair using the nonabsorbable prosthesis, polytetrafluoroethylene (Gore-Tex ${ }^{\circledR}$, W.L. Gore, Flagstaff, AZ), in a large series of survivors with appropriate long-term follow-up. Potential risk factors for recurrent $\mathrm{CDH}$ include diaphragm patch repair, abdominal wall patch repair, extracorporeal membrane oxygenation, and postoperative length of stay. ${ }^{2,4,11}$ Repair of a recurrent $\mathrm{CDH}$ is indicated in all symptomatic cases and in most asymptomatic patients because of the increased risk for bowel obstruction, intestinal ischemia, and respiratory compromise.

In 2007, our group began to routinely perform thoracoscopic repair in cases of recurrent $\mathrm{CDH}$ based on the premise that minimally invasive surgery (MIS) techniques may be particularly well suited in this patient population. These children usually have a larger working space as well as markedly improved pulmonary reserve compared with their neonatal counterparts. In addition, because most initial CDH repairs are still performed through a transabdominal approach, thoracoscopic recurrent $\mathrm{CDH}$ repair may be advantageous because of fewer adhesions within the operative field. This article presents our initial experience with thoracoscopic repair compared with open repair in children 
with recurrent $\mathrm{CDH}$ managed at a single pediatric tertiarycare referral center.

\section{Subjects and Methods}

\section{Patients}

A retrospective review of Bockdalek $\mathrm{CDH}$ patients treated postnatally at the University of Michigan C.S. Mott Children's Hospital (Ann Arbor, MI) between 1990 and 2011 was conducted under an approved institutional review board protocol (number 00040470). Of the $285 \mathrm{CDH}$ cases identified, $24(8.4 \%)$ had a recurrent $\mathrm{CDH}$ that was subsequently repaired. Data collected in recurrent $\mathrm{CDH}$ patients included side of defect, associated co-morbidities, and operative methods for all initial and recurrent repairs.

\section{Recurrent $\mathrm{CDH}$ repair}

Prior to 2007, all cases of recurrent CDH were repaired via laparotomy and thoracotomy. The techniques used in these open cases have been outlined elsewhere. ${ }^{12}$ Since 2007, the decision to undergo a thoracoscopic repair in the setting of recurrence was based on surgeon preference. Thoracoscopic repair was conducted in the full lateral decubitus position using three or four 3- or 5-mm ports for access to the chest similar to the operation described in neonates. ${ }^{13}$ Insufflation with carbon dioxide to $4-6 \mathrm{~mm} \mathrm{Hg}$ was used without single lung ventilation. Using a 5-mm, $30^{\circ}$ thoracoscope, all intrathoracic adhesions were lysed, and the abdominal viscera were reduced into the abdominal cavity. If there was no significant tension at the suture line, the hernia defect was closed primarily with several interrupted 2-0 nonabsorbable sutures. Otherwise, a patch was used to facilitate the repair. A small thoracostomy tube was placed at the conclusion of the procedure in the majority of cases.

\section{Statistical analyses}

Postoperative follow-up information, including chest radiographs, was obtained upon review of all operative and $\mathrm{CDH}$ clinic notes from the electronic medical record. Operative time, duration of mechanical ventilation, and hospital length of stay were evaluated as end points. Statistical analyses between the thoracoscopic repair group and open repair group were performed by the unpaired $t$ test and the twosided Fisher's exact test using the SPSS software package (IBM, Chicago, IL). Significance was defined as $P<.05$.
Patients converted from thoracoscopic to open repair $(n=2)$ were excluded in the comparative analyses.

\section{Results}

\section{Recurrent $\mathrm{CDH}$}

Of the 24 patients with recurrent $\mathrm{CDH}, 3$ had two recurrences, and 1 had four recurrences. Twenty $(83.3 \%)$ had left-sided recurrences. Ten $(41.7 \%)$ of the recurrences had a primary repair performed at the initial operation. Three $(12.5 \%)$ of the initial repairs were performed thoracoscopically. Fourteen (58\%) were supported on extracorporeal membrane oxygenation during the neonatal period. The median time from initial repair to the first recurrence was 9.0 months (range, 14 days -9.9 years). The mean weight at recurrent $\mathrm{CDH}$ repair was $9.5 \mathrm{~kg}$ (range, $2.9-25.9 \mathrm{~kg}$ ). Eighteen $(75 \%)$ of the 24 patients with recurrent $\mathrm{CDH}$ underwent open re-do repair, three of which were performed through a thoracotomy incision. The remaining $6(25 \%)$ children had an attempted thoracoscopic repair for recurrent $\mathrm{CDH}$ (Table 1).

\section{Thoracoscopic repair}

Although carbon dioxide insufflation was well tolerated in every patient, the first 2 children were converted to an open repair, resulting in an overall conversion rate of $33 \%$. Patient 1 was a 5-year-old child who was found to have significant intrathoracic adhesions at the time of his recurrent left $\mathrm{CDH}$ repair. These adhesions were presumably secondary to a prior parapneumonic effusion. Patient 2 was converted because of surgeon preference to perform a patch reconstruction for a large, right-sided defect through an open incision. Both children had their recurrent repairs performed through a subcostal laparotomy and did well postoperatively without complications.

The remaining 4 patients in the thoracoscopic repair group were successfully completed without conversion. Patient 3 was a 9-month-old infant who had had an open primary repair of his initial left $\mathrm{CDH}$ followed by multiple subsequent abdominal operations, including a Nissen fundoplication and gastrostomy tube. Given the anticipated difficulties in repairing his recurrence through a transabdominal approach, thoracoscopic repair was attempted. There was a recurrence identified at the midportion of the previous repair. The defect was closed primarily without tension using several nonabsorbable sutures and reinforced with two-ply porcine small

Table 1. Thoracoscopic Repair of Recurrent Congenital Diaphragmatic Hernia

\begin{tabular}{|c|c|c|c|c|c|c|}
\hline & Patient 1 & Patient 2 & Patient 3 & Patient 4 & Patient 5 & Patient 6 \\
\hline Defect side & Left & Right & Left & Left & Left & Left \\
\hline Initial repair & Patch & Muscle flap & Primary & Primary & Primary & Patch \\
\hline Length of stay, initial repair (days) & 32 & 40 & 43 & 25 & 32 & 145 \\
\hline Age at redo (months) & 60.1 & 6.5 & 8.1 & 9.3 & 12.6 & 16.1 \\
\hline Weight at redo $(\mathrm{kg})$ & 17.6 & 7.1 & 7.6 & 9.7 & 8.6 & 10.5 \\
\hline Conversion to open & Yes & Yes & No & No & No & No \\
\hline Re-do operating room time (minutes) & 609 & 247 & 146 & 94 & 294 & 232 \\
\hline Re-do repair with patch & No & Yes & Yes & No & No & Yes \\
\hline Length of stay, redo repair (days) & 6 & 3 & 4 & 1 & 4 & 6 \\
\hline Subsequent recurrence & No & No & No & No & No & No \\
\hline Follow up (months) & 99.4 & 28.5 & 41.3 & 28.2 & 22.2 & 14.3 \\
\hline
\end{tabular}



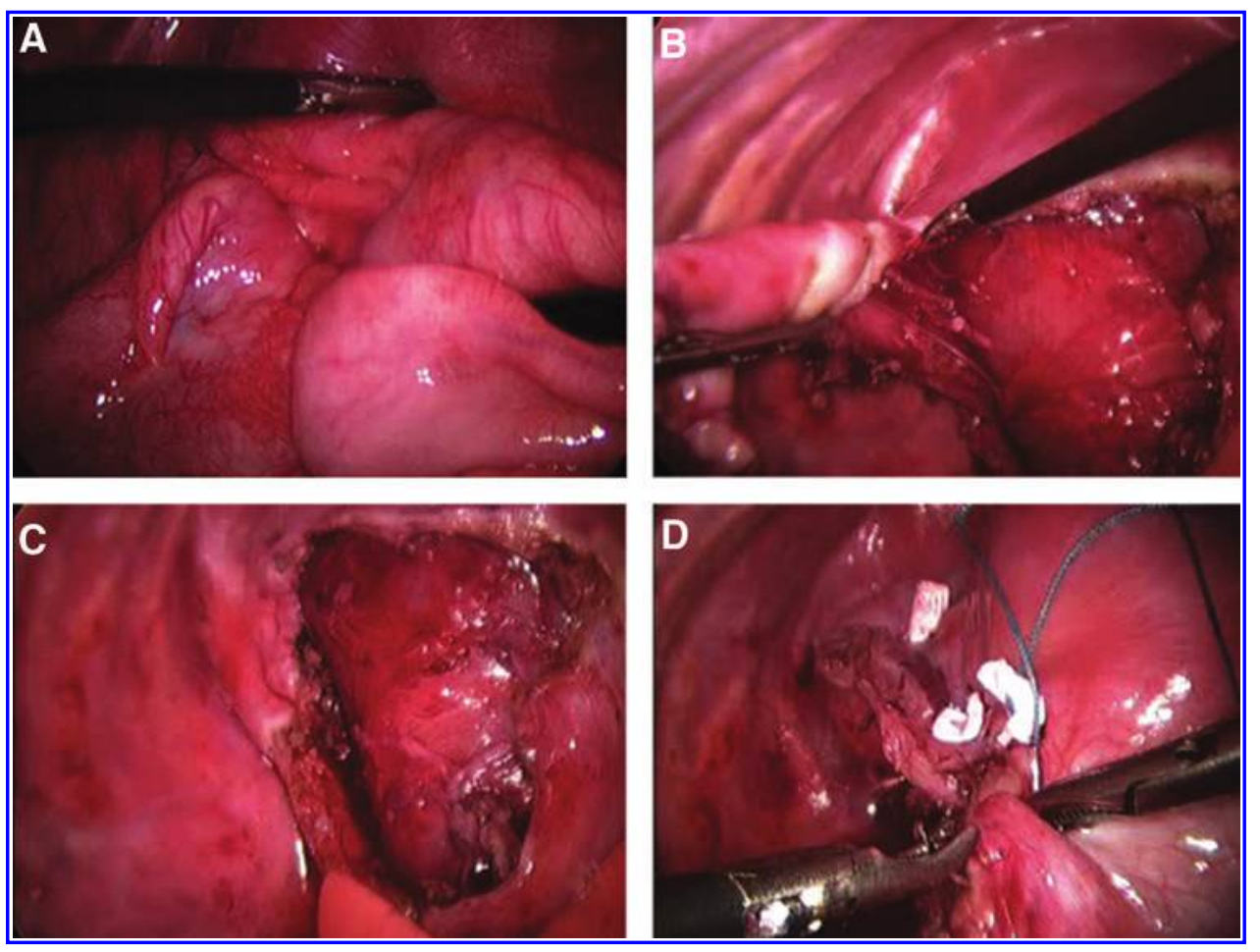

FIG. 1. Thoracoscopic repair of recurrent left congenital diaphragmatic hernia. (A) Reduction of abdominal viscera covered by a large hernia sac. (B) Excision of hernia sac. (C) Appearance of diaphragmatic rim. (D) Primary repair with interrupted, pledgetted sutures.

intestine submucosa $\left(\right.$ Surgisis $^{\circledR}$, Cook Technology, West Lafayette, IN). The child did well with minimal postoperative ileus and was discharged 4 days later.

Patients 4 and 5 each had large, left-sided eventration-type recurrences at the site of the original primary repair. After excision of the hernia sac, both of these recurrences were repaired in primary fashion (Fig. 1). Patient 5 also had a previously undiagnosed left extralobar pulmonary sequestration that was excised at the time of recurrent repair.

Patient 6 developed a recurrent left $\mathrm{CDH}$ following transabdominal repair of her initial defect with a porcine-based biological patch (Permacol ${ }^{\mathrm{TM}}$, Covidien, Mansfield, MA). She had a prolonged initial hospital course secondary to severe pulmonary hypertension requiring extracorporeal membrane oxygenation. She also had a chronic infection of an abdominal wall defect as well as severe gastroesophageal reflux, leading to multiple subsequent abdominal operations, including an extensive enterolysis, Nissen fundoplication, and gastrostomy tube. At the time of her recurrent $\mathrm{CDH}$ repair, thoracoscopy revealed herniated colon through a $1.5-\mathrm{cm}$ defect along the posterolateral aspect of the previous patch repair. The recurrence was closed primarily under moderate tension and further reinforced with Permacol as an onlay patch. Pericostal sutures were not required. On follow-up imaging studies, the patient was found to have a second, more medial recurrence of the $\mathrm{CDH}$ with evidence of herniated colon through the esophageal hiatus. Given the posteromedial location of the second recurrent defect, a laparoscopic paraesophageal hiatal hernia repair with re-do Nissen fundoplication was successfully performed.

Overall, the mean age at thoracoscopic recurrent $\mathrm{CDH}$ repair was 11.5 months (range, 8.1-16.1 months). Mean operative time was 191.5 minutes (range, 94-296 minutes). Blood loss was less than $15 \mathrm{~mL}$ in all cases, and there were no

Table 2. Comparison of Thoracoscopic and Open Repair of Recurrent Congenital Diaphragmatic Hernia

\begin{tabular}{lccc}
\hline & Thoracoscopic repair $(\mathrm{n}=4)$ & Open repair $(\mathrm{n}=18)$ & $\mathrm{P}$ value \\
\hline Age (months) & $11.5(8.1-16.1)$ & $25.3(0.07-120)$ & .774 \\
Weight $(\mathrm{kg})$ & $9.10(7.6-10.5)$ & $9.25(2.9-24.7)$ & $5(31.3 \%)$ \\
Patch repair at initial operation & $1(25 \%)$ & $209.3(63-474)$ & .965 \\
Operative time (minutes) & $191.5(94-296)$ & $9(50 \%)$ & .272 \\
Postoperative intubation & $1(25 \%)$ & $5.53(2-16)$ & .594 \\
Hospital length of stay (days) & $3.75(1-6)$ & $115.6(0.07-206.9)$ & .402 \\
Follow-up (months) & $26.5(14.3-41.3)$ & .010 \\
\hline
\end{tabular}

Results are presented as mean values with ranges in parentheses, unless otherwise indicated. Binomial variables were compared by the Fisher's exact test, and continuous variables were analyzed by the unpaired $t$ test. 


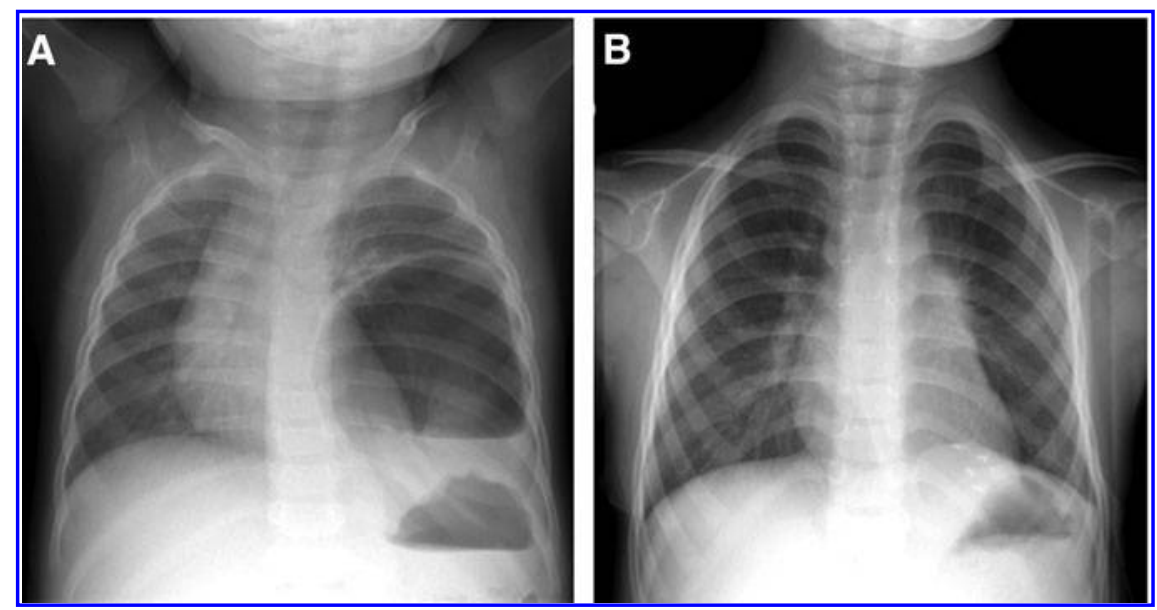

FIG. 2. Representative chest radiographs before and after thoracoscopic repair for recurrent congenital diaphragmatic hernia. (A) Preoperative chest radiograph of recurrent left congenital diaphragmatic hernia. (B) Postoperative chest radiograph at 2-year follow-up.

perioperative blood transfusions. All children were extubated within 24 hours following the repair. The mean hospital length of stay was 3.75 days (range, 1-6 days), and there were no complications. Compared with the open recurrent repair group, there were no significant differences in operative time, need for postoperative mechanical ventilation, or inpatient length of stay (Table 2). There were no deaths or subsequent recurrences following thoracoscopic repair after a mean follow up of 26.5 months (range, 14.3-41.3 months) (Fig. 2). In the open recurrent repair group, there were three perioperative deaths, and 3 children $(20 \%)$ developed a subsequent recurrence at a mean of 65.5 months following the first recurrent repair.

\section{Discussion}

Over the past decade, one of the most significant changes in the operative management of Bockdalek $\mathrm{CDH}$ has been the adoption of MIS techniques, particularly thoracoscopic repair. ${ }^{14-17}$ Proponents of MIS for CDH repair have touted several potential benefits compared with open repair, including less pain and incisional morbidity, reduced immunologic derangement and surgical stress, improved cosmesis, and faster recovery. ${ }^{17,18}$ It is interesting that there have only been a few large, prospective comparative studies to date between thoracoscopic repair and open repair for initial $\mathrm{CDH}$ defects. ${ }^{16,19}$ Among them, one of the few differences identified has been a higher short-term incidence of recurrent herniation following thoracoscopic repair. ${ }^{16}$ Although these data may be viewed as unfavorable for proponents of MIS, the long-term re-herniation rate following thoracoscopic $\mathrm{CDH}$ repair compared with open repair remains unknown. Moreover, the vast majority of these complications are likely secondary to technical failures, which tend to diminish over time as surgeon familiarity with thoracoscopic techniques increases. ${ }^{18}$

Despite these recent operative trends for the initial $\mathrm{CDH}$ repair, the recommended surgical approach for recurrent $\mathrm{CDH}$ remains traditional open surgery. ${ }^{12}$ We speculate that the continued reliance on open surgical techniques for recurrent herniation may be attributed to several factors, including the relative ease of placing a large prosthetic patch through an open incision as well as the perception that a technically demanding MIS procedure is likely to be even more difficult in the re-operative setting. ${ }^{20,21}$ Although most surgeons advocate a laparotomy incision as the best approach for recurrent repair, some prefer a thoracotomy incision in selected situations, particularly in right-sided hernias or when significant intra-abdominal adhesions are anticipated. ${ }^{22}$ Subsequent recurrences after recurrent open repair have never been carefully studied, but our own rate of $20 \%$ is consistent with that documented by others in the literature.

In this study, we reviewed our early data on thoracoscopic repair for recurrent $\mathrm{CDH}$, a relatively unexplored area in pediatric thoracic surgery. There is certainly a learning curve associated with MIS re-do procedures, but operative times were not significantly different than for those who underwent open recurrent repair. More important is that there were no complications, added morbidity, or subsequent recurrences in the thoracoscopic group after a mean follow-up period of over 2 years. Our experience concurs with that of Keijzer et al., ${ }^{23}$ who also recently reported successful repair of five $\mathrm{CDH}$ recurrences using similar thoracoscopic techniques. Unfortunately, the relative benefits of MIS were unclear from that study because there were no data provided regarding patient age, operative times, or other outcome parameters compared with those undergoing open repair.

There are many reasons why thoracoscopic repair may be the preferred operative approach for recurrent $\mathrm{CDH}$. Thoracoscopy provides the surgeon with excellent visualization of the recurrent defect, enabling safe reduction of the intraabdominal viscera and subsequent diaphragmatic reconstruction comparable or superior to what could be achieved through an open incision. In other cases, thoracoscopic repair obviates the need for an extensive enterolysis that may be required if the procedure was conducted through a previous laparotomy incision. Indeed, 2 of our patients with recurrent $\mathrm{CDH}$ had indwelling gastrostomy tubes that would have made the transabdominal approach even more tedious and difficult. Finally, thoracoscopy avoids many of the potential long-term musculoskeletal morbidities associated with a thoracotomy in young children. ${ }^{24}$ 
Based on this series, the child with a failed initial primary repair appears to be an excellent candidate for thoracoscopic recurrent $\mathrm{CDH}$ repair. Because these patients often present with an eventration rather than a bona fide hernia, the insufflation pressure within the thorax can be particularly helpful in reducing the defect and in defining the rim of the diaphragm. Children who underwent a previous patch repair are also potential candidates for thoracoscopic repair for recurrence because many of these defects are quite small, occur laterally, and are therefore relatively straightforward to repair by patch augmentation. As shown in Patient 4, prior thoracoscopic repair should not be viewed as a contraindication to thoracoscopic recurrent $\mathrm{CDH}$ repair.

Nonetheless, not all patients are good candidates for thoracoscopic repair of an initial $\mathrm{CDH}$ defect, and the same holds true in cases of $\mathrm{CDH}$ recurrence. The ideal operative approach for recurrent $\mathrm{CDH}$ should be individualized depending on multiple factors, including the patient's clinical status, precise location of the recurrence, and need for concurrent procedures such as a Nissen fundoplication. Those with persistent pulmonary hypertension or inadequate lung reserve are less unlikely to tolerate thoracoscopy for recurrent defects. More recently, there have been some early data to suggest that thoracoscopic repair may lead to perioperative respiratory acidosis and other deleterious effects, including decreased cerebral oxygenation, in this vulnerable patient population. ${ }^{25}$ Children with a history of an infectious process or a chylothorax in the ipsilateral hemithorax, as seen in Patient 1, may also not be ideal candidates for thoracoscopic repair because of the likely formation of dense pleural adhesions that can severely compromise visualization, leading to subsequent lung parenchyma injury as well as prolonged operative times. Moreover, as shown in Patient 6 and as suggested by others, ${ }^{12}$ if a component of the recurrence manifests as a paraesophageal hernia along the posteromedial aspect of the diaphragm, this can be a very difficult area to visualize and repair using thoracoscopic techniques. Such patients with large posteromedial recurrences are probably best served by a traditional, transabdominal open approach. Given that none of the patients in our series had large recurrent defects, the utility of the thoracoscopic approach for hernias requiring extensive patch reconstruction has yet to be determined.

Despite our favorable results demonstrating the feasibility of thoracoscopic repair in selected patients with recurrent $\mathrm{CDH}$, this was a retrospective, single-institution study of a small number of patients. Longer follow-up is needed to fully assess the long-term durability of these repairs. Further experience with thoracoscopic recurrent $\mathrm{CDH}$ repair, perhaps with collaboration with other pediatric referral centers, will help to critically evaluate and further define the appropriate place of MIS in this challenging patient population.

\section{Acknowledgments}

The authors wish to thank Mr. Robert A. Drongowski for his statistical assistance.

\section{Disclosure Statement}

No competing financial interests exist.

\section{References}

1. Rowe DH, Stolar CJ. Recurrent diaphragmatic hernia. Semin Pediatr Surg 2003;12:107-109.

2. Moss RL, Chen CM, Harrison MR. Prosthetic patch durability in congenital diaphragmatic hernia: A long-term follow-up study. J Pediatr Surg 2001;36:152-154.

3. Nasr A, Struijs MC, Ein SH, et al. Outcomes after muscle flap vs prosthetic patch repair for large congenital diaphragmatic hernias. J Pediatr Surg 2010;45:151-154.

4. Fisher JC, Haley MJ, Ruiz-Elizalde A, et al. Multivariate model for predicting recurrence in congenital diaphragmatic hernia. J Pediatr Surg 2009;44:1173-1179; discussion 1179_ 1180.

5. Laituri CA, Garey CL, Valusek PA, et al. Outcome of congenital diaphragmatic hernia repair depending on patch type. Eur J Pediatr Surg 2010;20:363-365.

6. Lally KP, Paranka MS, Roden J, et al. Congenital diaphragmatic hernia. Stabilization and repair on ECMO. Ann Surg 1992;216:569-573.

7. Jancelewicz T, Vu LT, Keller RL, et al. Long-term surgical outcomes in congenital diaphragmatic hernia: Observations from a single institution. J Pediatr Surg 2010;45:155-160; discussion 160.

8. Mitchell IC, Garcia NM, Barber R, et al. Permacol: A potential biologic patch alternative in congenital diaphragmatic hernia repair. J Pediatr Surg 2008;43:2161-2164.

9. Grethel EJ, Cortes RA, Wagner AJ, et al. Prosthetic patches for congenital diaphragmatic hernia repair: Surgisis vs GoreTex. J Pediatr Surg 2006;41:29-33; discussion 29-33.

10. Riehle KJ, Magnuson DK, Waldhausen JH. Low recurrence rate after Gore-Tex/Marlex composite patch repair for posterolateral congenital diaphragmatic hernia. J Pediatr Surg 2007;42:1841-1844.

11. Hajer GF, vd Staak FH, de Haan AF, et al. Recurrent congenital diaphragmatic hernia; which factors are involved? Eur J Pediatr Surg 1998;8:329-333.

12. Hayward ML, Fauza DO, Wilson JM. Complications of CDH repair and recurrent $\mathrm{CDH}$. In: Teich S, Caniano DA (eds). Reoperative Pediatric Surgery. Totowa, NJ: Humana Press, 2008, pp. 139-156.

13. Kim AC, Bryner BS, Akay B, et al. Thoracoscopic repair of congenital diaphragmatic hernia in neonates: Lessons learned. L Laparoendosc Adv Surg Tech A 2009;19:575-580.

14. Arca MJ, Barnhart DC, Lelli JL Jr, et al. Early experience with minimally invasive repair of congenital diaphragmatic hernias: Results and lessons learned. J Pediatr Surg 2003;38: 1563-1568.

15. Yang EY, Allmendinger N, Johnson SM, et al. Neonatal thoracoscopic repair of congenital diaphragmatic hernia: Selection criteria for successful outcome. J Pediatr Surg 2005; 40:1369-1375.

16. Tsao K, Lally PA, Lally KP. Minimally invasive repair of congenital diaphragmatic hernia. J Pediatr Surg 2011;46: 1158-1164.

17. Gourlay DM, Cassidy LD, Sato TT, et al. Beyond feasibility: A comparison of newborns undergoing thoracoscopic and open repair of congenital diaphragmatic hernias. I Pediatr Surg 2009;44:1702-1707.

18. Liem NT, Nhat LQ, Tuan TM, et al. Thoracoscopic repair for congenital diaphragmatic hernia: Experience with 139 cases. J Laparoendosc Adv Surg Tech A 2011;21:267-270.

19. Lao OB, Crouthamel MR, Goldin AB, et al. Thoracoscopic repair of congenital diaphragmatic hernia in infancy. $\mathrm{J}$ Laparoendosc Adv Surg Tech A 2010;20:271-276. 
20. Lansdale N, Alam S, Losty PD, et al. Neonatal endosurgical congenital diaphragmatic hernia repair: A systematic review and meta-analysis. Ann Surg 2010;252:20-26.

21. Gander JW, Fisher JC, Gross ER, et al. Early recurrence of congenital diaphragmatic hernia is higher after thoracoscopic than open repair: A single institutional study. J Pediatr Surg 2011;46:1303-1308.

22. Saltzman DA, Ennis JS, Mehall JR, et al. Recurrent congenital diaphragmatic hernia: A novel repair. I Pediatr Surg 2001;36: 1768-1769.

23. Keijzer R, van de Ven C, Vlot J, et al. Thoracoscopic repair in congenital diaphragmatic hernia: Patching is safe and reduces the recurrence rate. J Pediatr Surg 2010;45: 953-957.

24. Lawal TA, Gosemann JH, Kuebler JF, et al. Thoracoscopy versus thoracotomy improves midterm musculoskeletal status and cosmesis in infants and children. Ann Thorac Surg 2009;87:224-228.

25. Bishay M, Giacomello L, Retrosi G, et al. Decreased cerebral oxygen saturation during thoracoscopic repair of congenital diaphragmatic hernia and esophageal atresia in infants. I Pediatr Surg 2011;46:47-51.

Address correspondence to: Shaun M. Kunisaki, MD Section of Pediatric Surgery University of Michigan

C.S. Mott Children's Hospital 1540 East Hospital Drive, SPC 4211 Ann Arbor, MI 48109

E-mail: shaunkun@umich.edu 